in the higher vertebrates is " heat," but in very few is this accompanied by a discharge of blood, and where there is a slight staining the proportion of red blood corpuscles to white is very small, as in those macaque monkeys observed by Heape of Cambridge. In the case of "Johannah," the famous chimpanzee, whose menstrual history I have recorded elsewhere, I made a careful microscopical examination of the menstrual discharge and satisfied myself that the red corpuscles were comparatively small in number, though enough to tinge distinctly and to stain red the monthly flow; still, all these were under the artificial surroundings of captivity. Not only the attendant pain and copious loss in woman but the general and nervous disturbances occurring at these times are suggestive of a departure from the originally normal line. The amenorrhcea accompanying lactation is often regarded by women as a welcome condition of security from impregnation. This we know to be an error, for ovulation is proceeding with its accustomed regularity and impregnation may, and often does, take place. Furthermore, the development of the endometrium every month may be safely regarded as the preparation of the uterus for the implantation of an impregnated ovum and the menstruation may be considered as evidence of a failure of these anticipations.

It is quite likely that primitive woman never menstruated, at any rate as we know menstruation to-day, and there is reason to believe that this phenomenon, this heritage of woman, of which she is not proud and for which she is not grateful, has assumed its present proportions as a result of the almost universal practice, begun in the earliest ages, of restraining the excessive uberty of our primeval ancestors.

Curzon-street, W.

\section{A CASE OF}

\section{CHRONIC INTESTINAL OBSTRUCTION WITH PERFORATION OF THE SIGMOID FLEXURE.}

\section{BY GEORGE A. CLARKSON, F.R.C.S. EYG.}

THE notes of the following case present one or two points of considerable interest. At the time of the first operation the history and the age of the patient led me to believe that I was dealing with a stricture of some part of the colon due to growth but the distension of the small intestine and cæcum was so great and the call for immediate drainage of the distended bowel was so urgent that no prolonged examination of the colon could be made. The subsequent sloughing and perforation of the sigmoid flexure probably occurred at the seat of obstruction, yet here no cause was obvious to account for the condition found. The gut, though in a condition little short of gangrene, was apparently not invaded by growth and the fact that no fæcal matter was found in it rather excluded the idea that retention of a scybalous mass of fæces at that point had given rise to inflammatory softening and perforation of its coats.

The patient, a woman, aged 52 years, had suffered from disease of the outer surface of the acetabulum for 12 years and the sinuses on the outer side of the right hip had healed after a free exploration and scraping in May, 1903. In the spring of 1904 she began to be subject to occasional attacks of vomiting, accompanied by abdominal pain, distension, and constipation, but these attacks were relieved from time to time by aperients and enemata. On May 9th she was seized with a severer attack than usual. The bowels were at first relieved and the sickness would stop for 12 hours at a time but invariably returned again. The patient was kept in bed, put on a fluid diet, and saline aperients and enemata were given with some result. A rectal examination revealed no stricture within reach of the finger nor could anything be felt through the abdominal walls to localise the seat of obstruction. On the 15th the symptoms became urgent, the vomiting being persistent and copious but not offensive in smell, the abdominal distension became very great, and there was obviously more or less complete obstruction. Enemata given through a long rectal tube produced no effect, so the patient was removed without delay to a surgical home. The opera. tion was carried out in the evening. The abdomen was opened in the middle line below the umbilicus. Enormous distension of the small intestine and cæcum was met with and as the transverse and descending colon appeared less distended and the indication to drain the bowel with as little delay as possible was urgent the wound was closed and another small incision was made over the cæcum, which was drawn out, opened, and a Patul's tube tied in. Following the operation there was a little vomiting of a stercoraceous character but the caccal opening gave great relief and the acute symptoms subsided. At the end of a fortnight the patient was able to be removed home. Enemata were subsequently given from time to time and occasionally brought away small scybalous masses.

On the afternoon of June 11th the patient was suddenly taker ill with intense pain in the left inguinal region and when I saw her was extremely collapsed, with drawn features, cold extremities, and thready pulse. Subcutaneous injections of strychnine with a little morphine were given and she gradually rallied, symptoms of an acute localised peritonitis quickly manifesting themselves. A diagnosis of intestinal perforation was made and on the 15 th an incision similar to that for inguinal colotomy was made over the area of pain and resistance. On opening the abdomen some very foul-smelling pus welled up and the abscess cavity having been thoroughly swabbed out the sigmoid flexure was seen plastered over with thick lymph and with a perforation on its anterior surface of the size of a shilling. The coats of the bowel were softened, black, and gangrenous and in bringing it forward it tore almost across. The wound was therefore enlarged in an upward direction and the bowel was slit up with scissors till its walls appeared unaffected by the changes below and would allow of its being stitched to the skin which was then done. The whole condition seemed to be an acutely inflammatory one. The abscess cavity was again swabbed out and then packed with iodoform gauze, an indiarubber tube of large calibre being passed into the colon.

The patient stood the operation well. The discharge was most offensive for some days but the cavity contracted quickly and at the end of a month the opening differed but little from an ordinary colotomy opening and it acted well. As the crcal opening became an annoyance to the patient when she began to get about again owing to the liquidity of the discharge, on August 11th, under ether, the mucous membrane was separated from the skin and the opening closed. At the time of writing (March, 1905) the patient finds no difficulty in controlling the artificial anus and she is able to get about out of doors and to resume her ordinary domestic duties at home.

Leicester.

\section{Clinital 睯otes:}

MEDICAL, SURGICAL, OBSTETRICAL, AND THERAPEUTICAL.

\section{A NOTE ON THE ABDOMINAL INCISIONS FOR THE EXPLORATION OF THE APPENDIX AND PER-} FORATED ULCER OF THE STOMACH.

\section{By Curnbert S. Wallace, M.B., B.S. LoNd.} F.R.C.S. ENG.,

SURGEON TO OUT-PATIENTS AT ST, THOMASS HOSPITAL; ASSISTANT SURGEON TO THE EAST LONDON HOSPITAL FOR CHILDREN.

The incision for exploration of the appendix.-The two abdominal incisions that are most in vogue for removal of the appendix-namely, that of McBurney and that through the rectus sheath-have their advantages and disadvantages. The McBurney incision is cramped and if difficulties are encountered gives poor access even if strongly spanned by assistants. It is, on the other hand, easily and quickly closed. The rectus sheath incision gives a good view and can be enlarged without difficulty to any extent; it is, however, often difficult to close owing to the tendency of the layers behind the rectus muscle to retract towards the iliac crest. The following modification seems to combine some of the advantages of both incisions. The rectus sheath is entered towards its outer side by the usual vertical incision. The muscle is then retracted inwards and the posterior wall of the sheath is exposed. As a rule two nerves are seen running transversely inwards about one inch apart. The mid-point between these is selected and the 